\title{
Effects of Acrylamide and Vitamin C on Histological Changes and Stereological Parameters of Cerebellum in Rat Offsprings
}

Hengameh Dortaj, Maryam Yadegari, Mohammad Hosseini Sharif Abad, Abolghasem Abbasi Sarcheshmeh, Morteza

\section{Anvari* $^{*}$}

Department of Biology \& Anatomical Sciences, Faculty of Medicine, Shahid Sadoughi University of Medical Sciences, Yazd, Iran.

\section{A BSTRACT}

Introduction: Acrylamide (ACR) is a substance chemical used in industrial and laboratory procedures. Acrylamide according to the method of cooking foods are increasingly used and its adverse effects on multiple organ systems have been described sporadically in the literature. The aim of this study was to investigate the effects of prenatal (maternal) ACR consumption on the physical development and the changes of cerebellar volume in the rat embryo. Materials and Methods: Female pregnant Wistar rats were orally administered $10 \mathrm{mg} / \mathrm{kg}$ ACR and/or $200 \mathrm{mg} / \mathrm{kg}$ vitamin C (vit C) solution. Pregnant rats were sacrificed on the $15^{\text {th }}$ day of gestation and mother's weight was measured. After that, their fetuses were taken out and were evaluated for fetus number and weight, crown-rump length (CRL) and cerebellar development. A total of 100 sections were cut through the entire cerebellum. From these sections, approximately 10 equidistant sections were systematic uniformly random sampled with an initial random start and stained with hematoxylin-eosin for volume estimation. The cerebellar volume was estimated by micro-projection and cavaliers' method. Results: The results showed that ACR caused a significant reduction in mother's weight and volume of cerebellum as well as in the number of fetus. Histological and stereological examinations revealed that the cerebellar volume was decreased in ACR and ACR+vit C groups vs control animals. Conclusion: ACR exhibits a harmful effect on the development of the brain, which may be prevented by administration of vit $\mathrm{C}$.

\section{Key words:}

1. Cerebellar Diseases

2. Acrylamide

3. Histology

4. Embryonic Structures

5. Rats

* Corresponding Author: Morteza Anvari

E-mail:moanvari@yahoo.com 


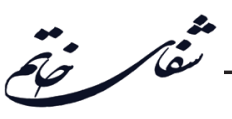

اثرات آكريل آميدو ويتامين Cبر تغيير ات بافتى شاخص هاى استريولورى مخجه در جنين هاى موش صحرايى

هنًَامه در تاج، مريم يادًَارى، محمد حسينى شريف آباد، ابوالقاسم عباسى سر جشمه، مرتضى انورى"

كروه علوم تشريح و بيولوزى، دانشكده يزشكى، دانشكاه علوم يزشكى شهيد صدوقى، يزد، ايران

كليد وازهها:

1. بيمارىهاى مخجه ז. آكريل آميد

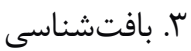
f ه. موش صحرايى
مقدمه: آكريلآميد يك ماده شيميايى است كه در صنعت و فرايندهاى آزمايشگاهى كاربرد دارد.

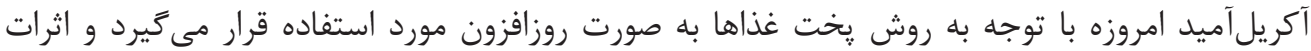

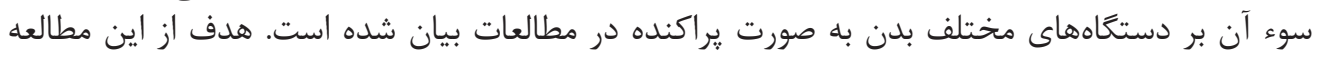

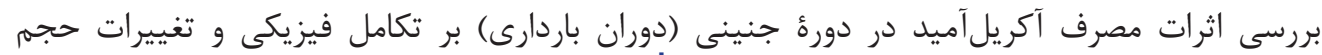

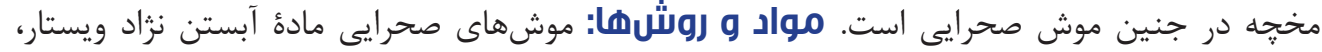

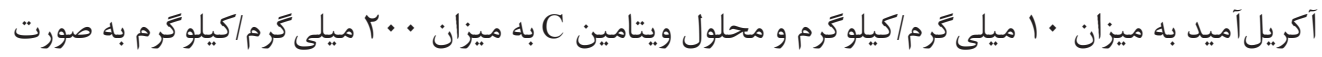

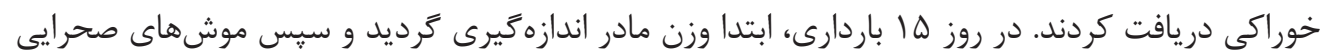

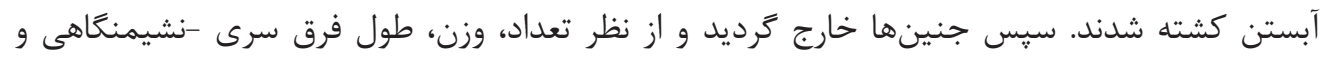

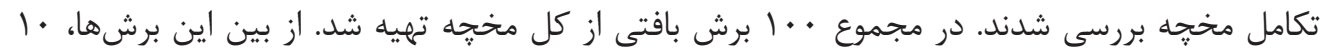

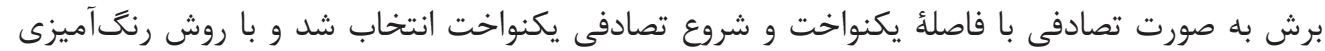

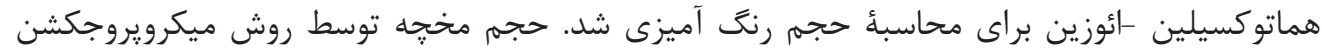

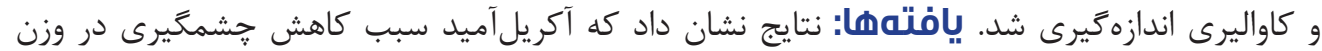

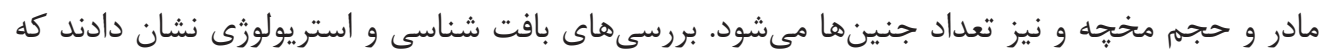

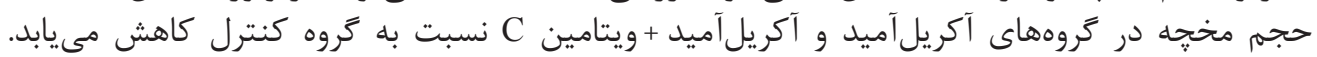

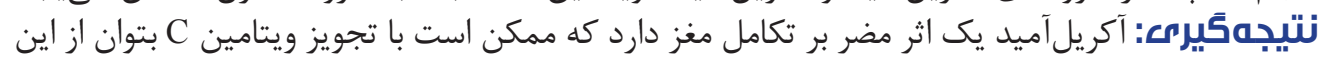

" نويسنده مسئول: مرتضى انورى : آدرس الكترونيكى: moanvari@yahoo.com 


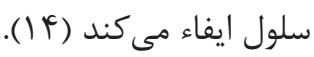

حجم كلى كورتكس، ماده سفيد، مساحت سطح مخحٍه، تراكم

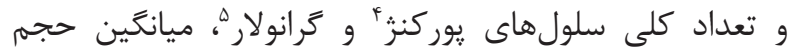

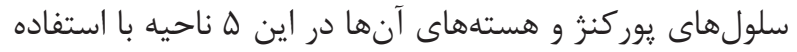

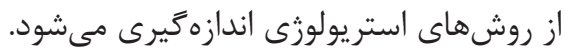

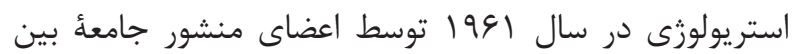

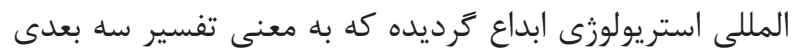

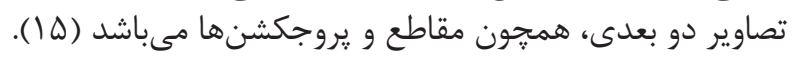

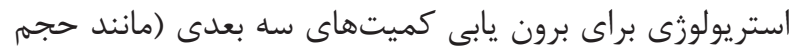

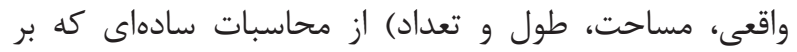

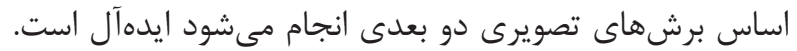

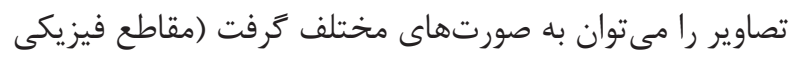

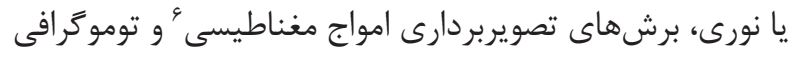

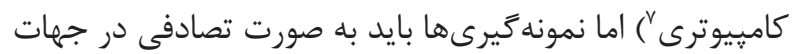

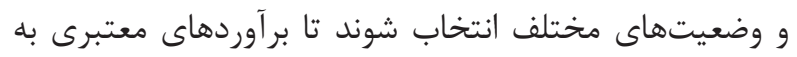

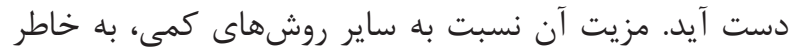

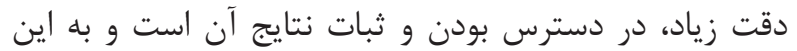

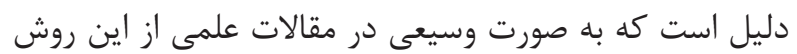

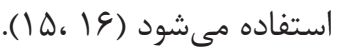

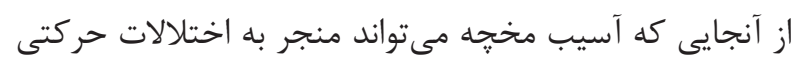

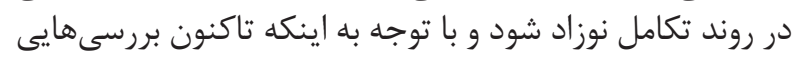

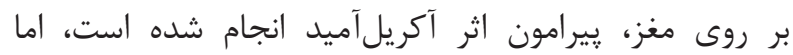

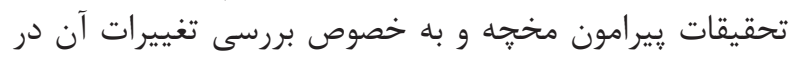

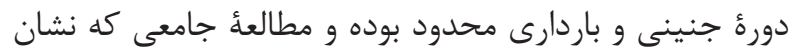

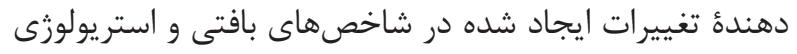

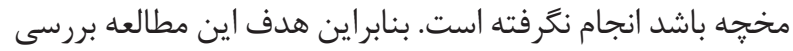

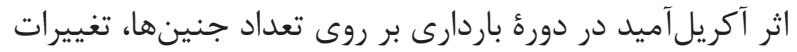

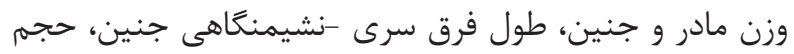

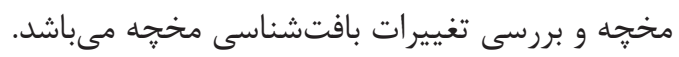

مواد و روشها

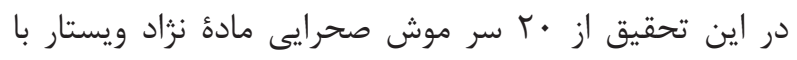

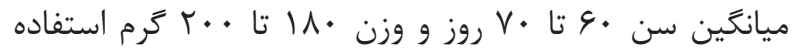

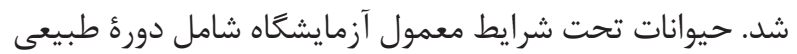

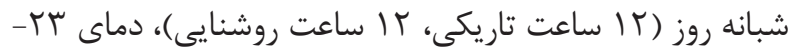

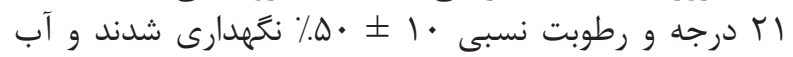

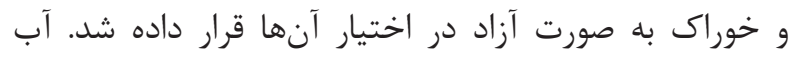

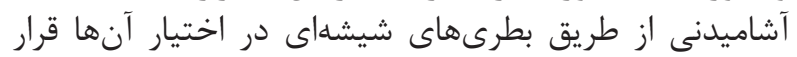

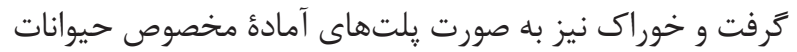

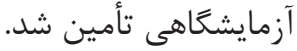

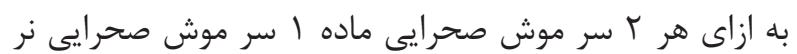

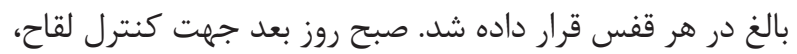

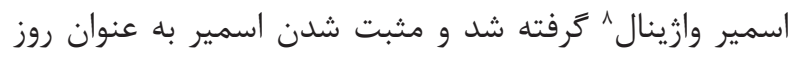

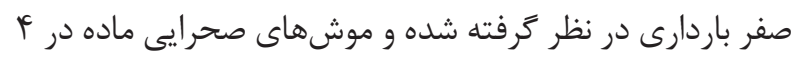
كروه ينج تايى به صورت زير تقسيمبندى شرف شدند

${ }^{1}$ Acrylamide

${ }^{2} \mathrm{H}_{2} \mathrm{O}_{2}$

${ }^{3}$ Ascorbic acid

${ }^{4}$ Purkinje cell

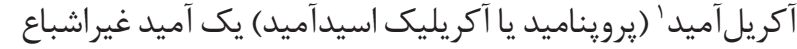
داراى فرمول شيميايى (برئ

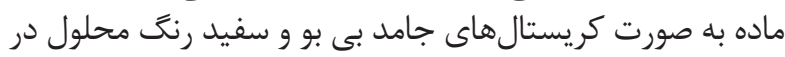

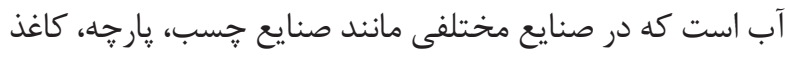

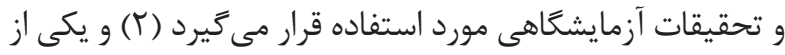

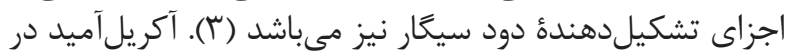

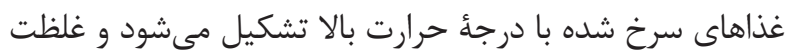

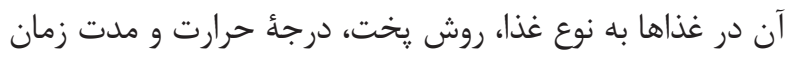

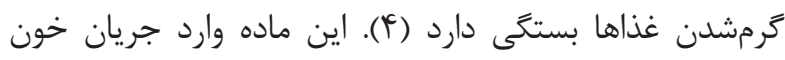

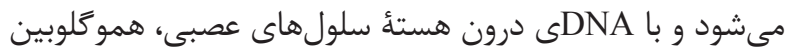

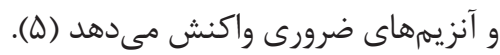

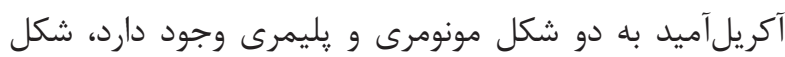

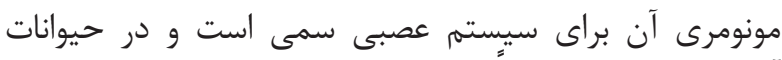

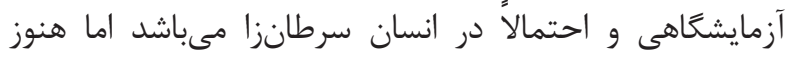

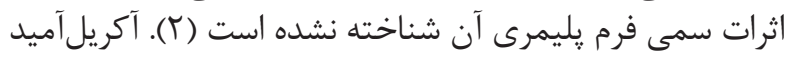

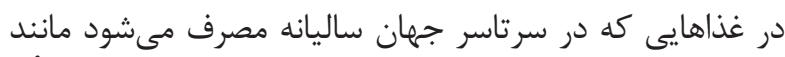

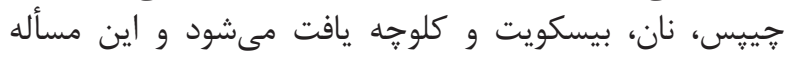

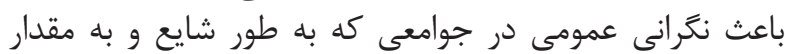

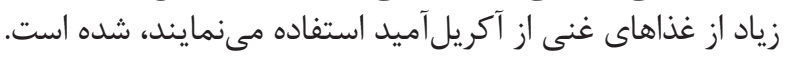

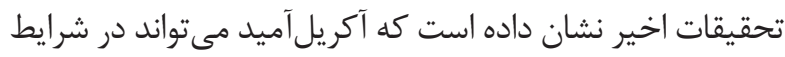

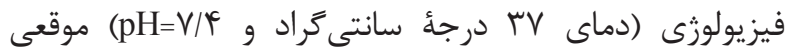

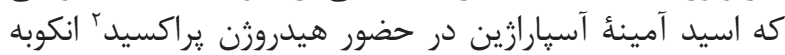

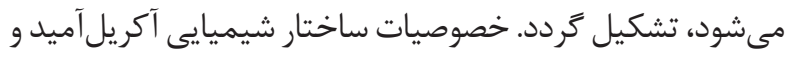

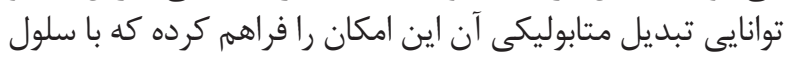

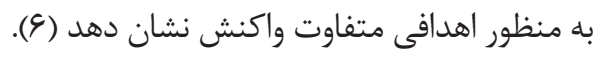

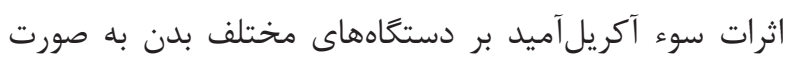

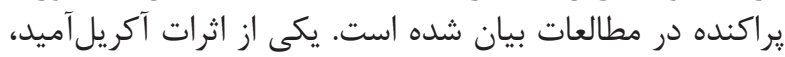

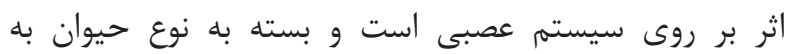

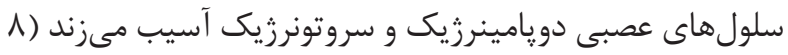

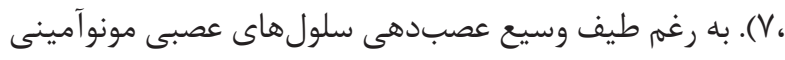

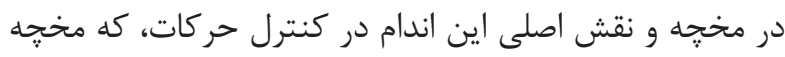

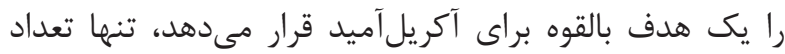

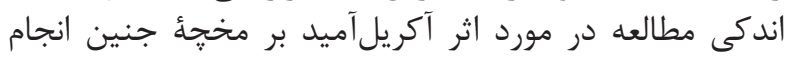

شده است (9). (9) (9)

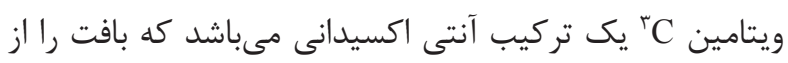

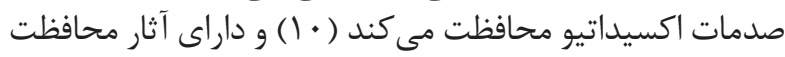

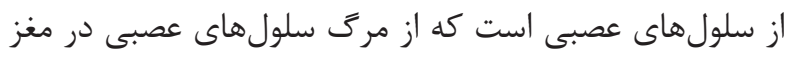

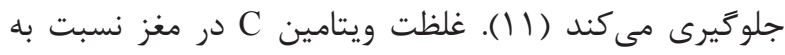

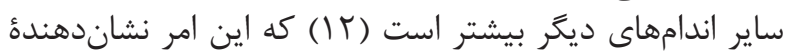

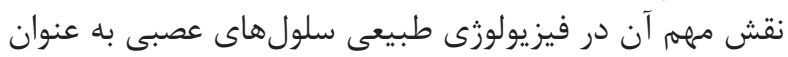

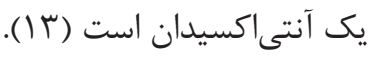

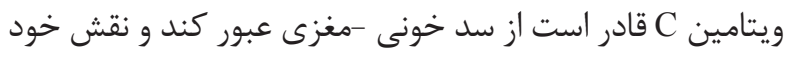

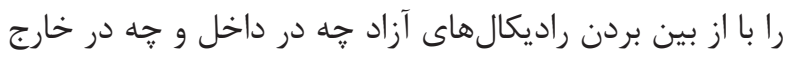

${ }^{5}$ Granular cell

${ }^{6}$ Magnetic Resonance Imaging (MRI)

${ }^{7}$ Computed Tomography (CT)

${ }^{8}$ Vaginal smear 


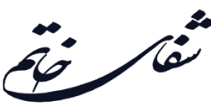

برش و (pala؛ مساحت اطراف هر نقطه مىباشد (1). براى به دست آوردن مساحت اطراف هر نقطه ابتدا طول شبكه

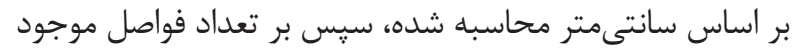

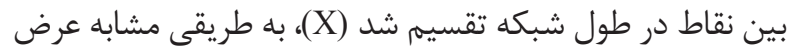

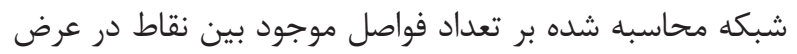

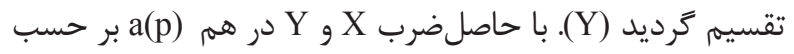

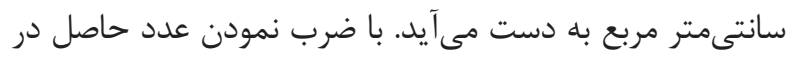

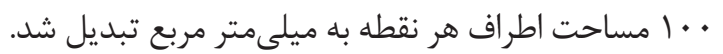

$$
\text { تجزيه و تحليل آمارى }
$$

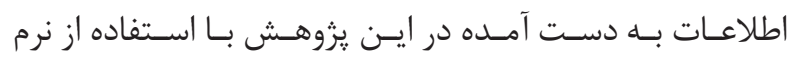

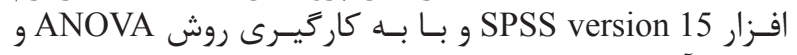

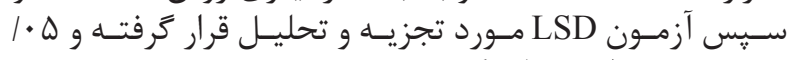
•••

يافته ها

نتايج، اختلاف معنادارى را بين گروههاى كنترل و آزمايش در مصر

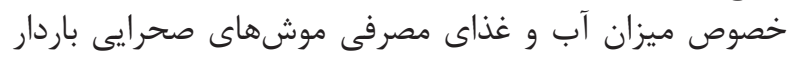

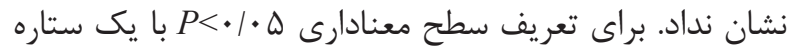

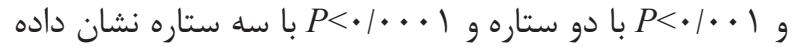

شده است.

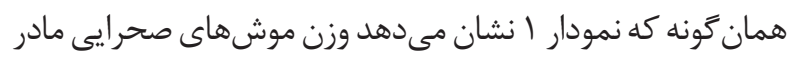

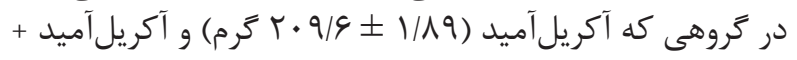

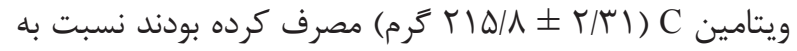

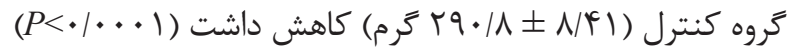

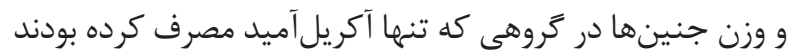

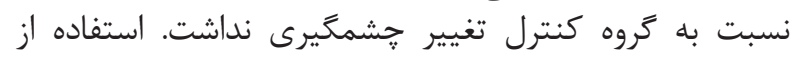

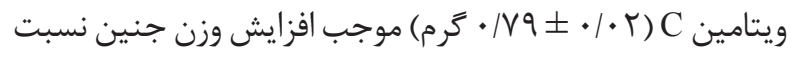
به كروه كنترل (| • (

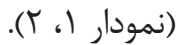

$$
\text { • ََروه الف: كنترل (مصرف آب معمولى). }
$$

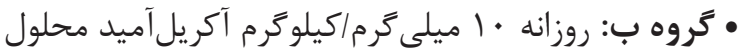

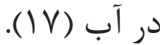

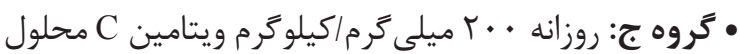

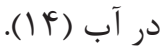

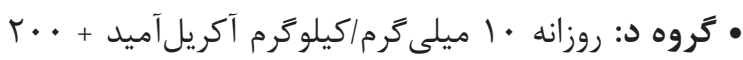
ميلى

بعد از كذشت ها روز از شروع باردارى، موشهاى صحرايى

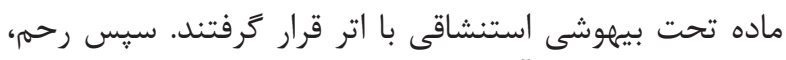

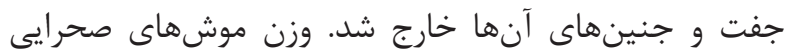

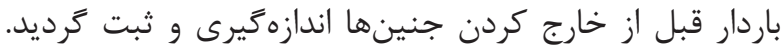

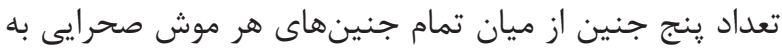

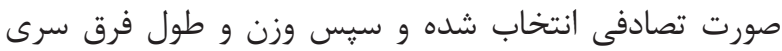

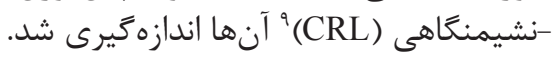

تمام جنينها براى فيكس شدن به مدت سه هفته در محلول

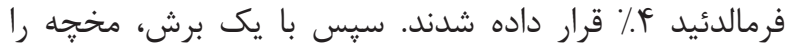

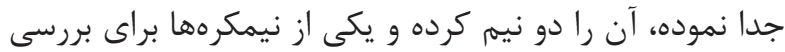

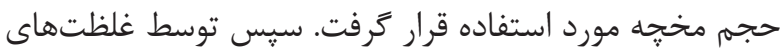

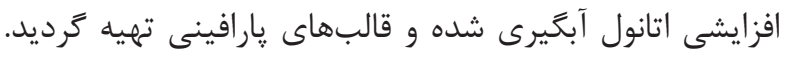

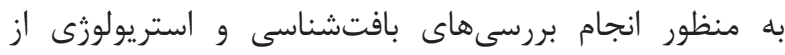

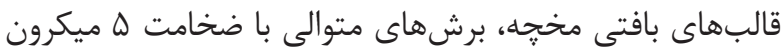

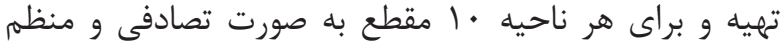

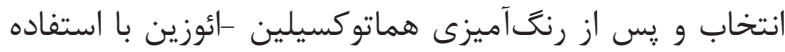

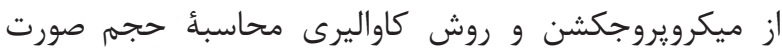

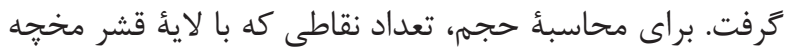

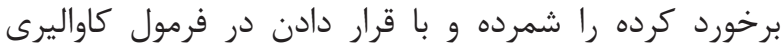

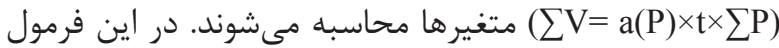
\&P

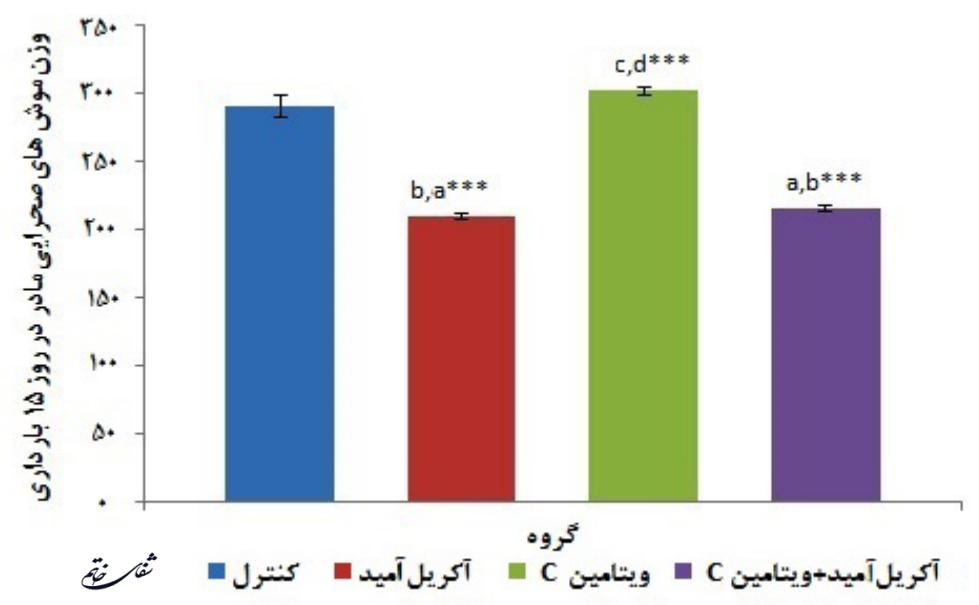

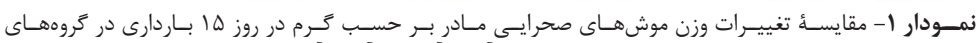

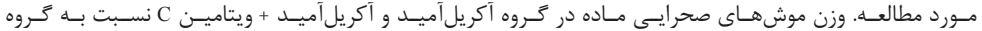

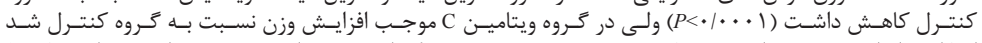

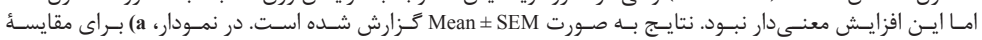

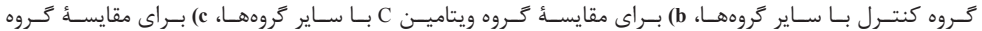

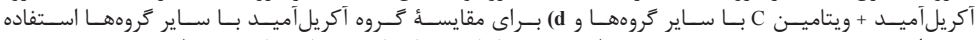

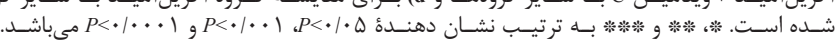

${ }^{9}$ Crown-Rump Length 


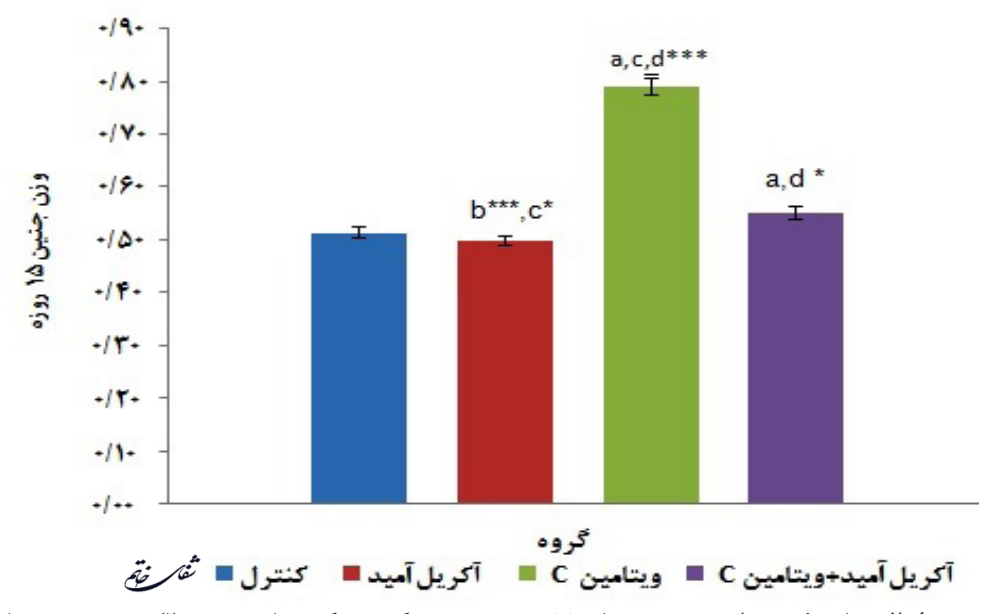

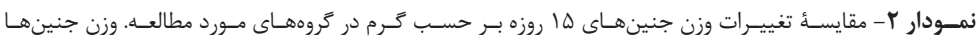

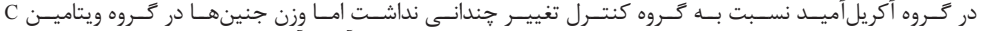

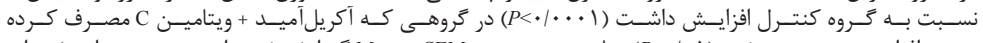

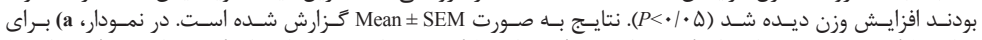

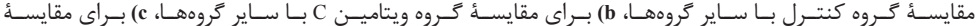

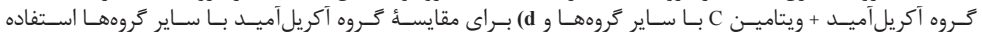

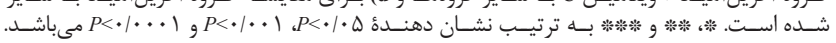

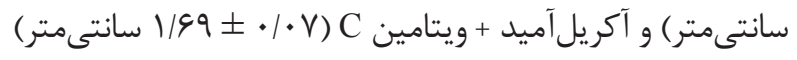

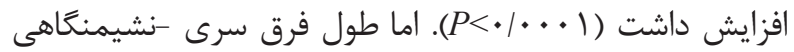

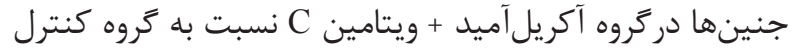

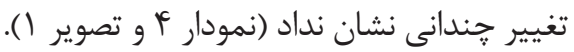

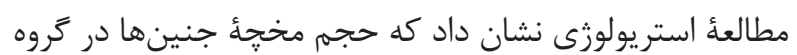

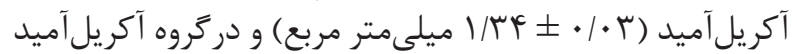

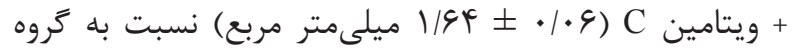

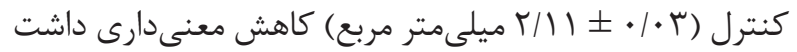

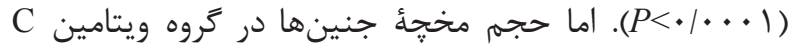

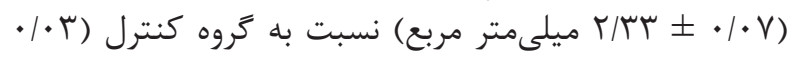

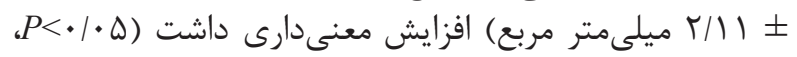

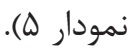

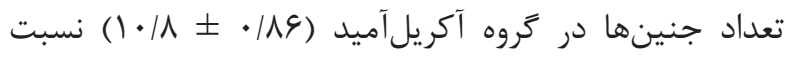

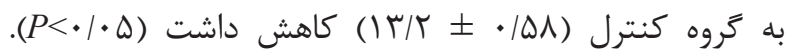

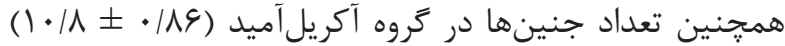

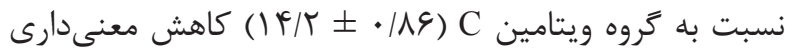

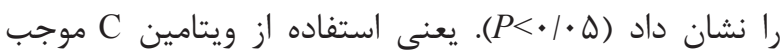

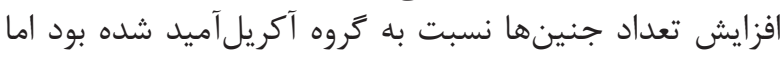

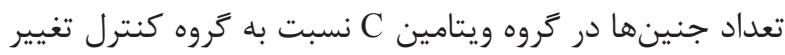
جندانى نداشت (نمودار بن). طول فرق سرى -نشيمنغاهى جنينها در كروه آريل آريد

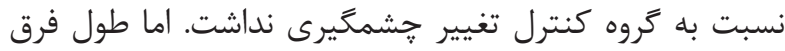

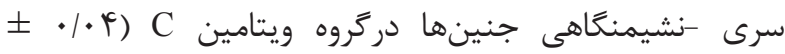

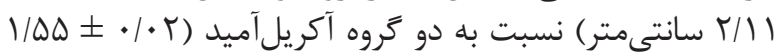

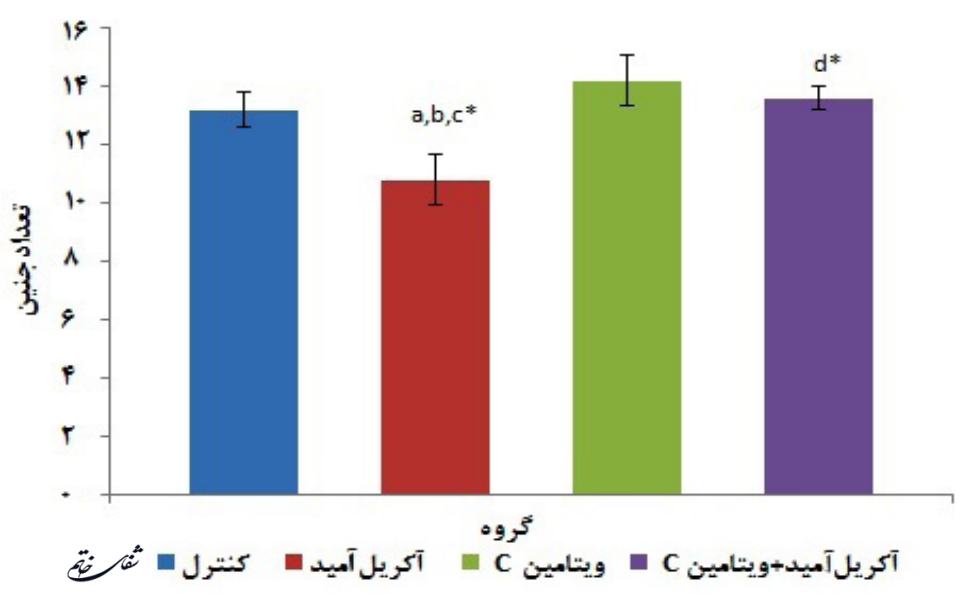

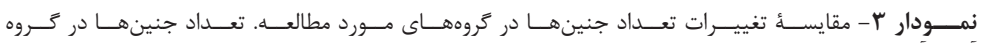

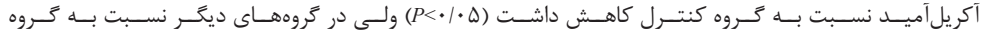

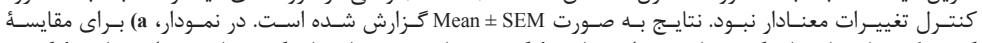

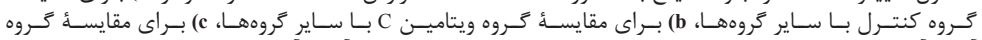

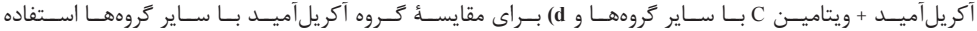

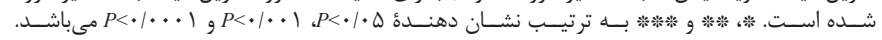




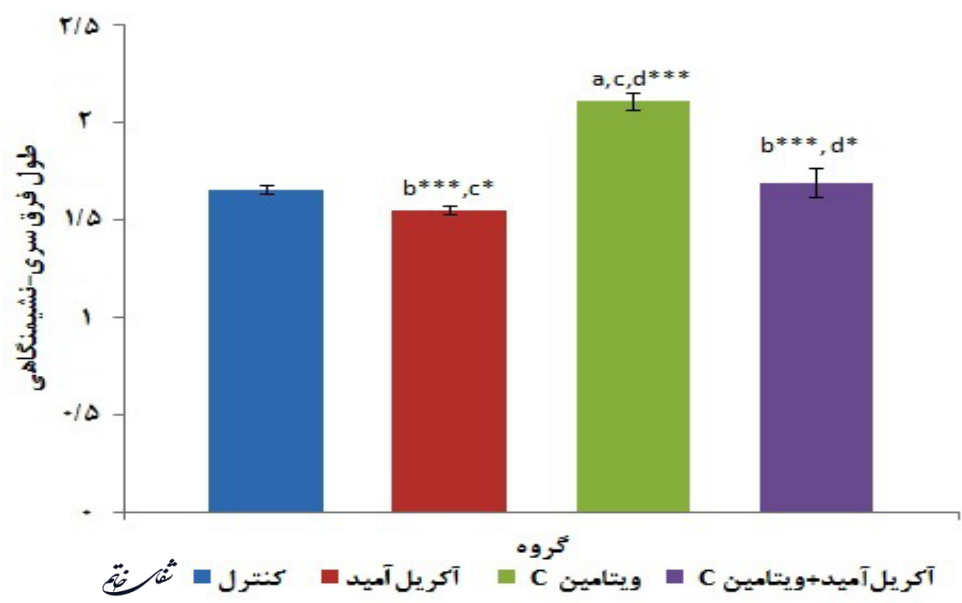

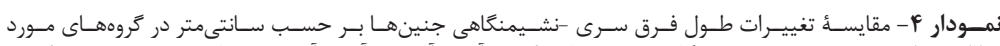

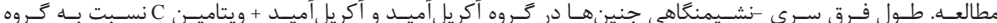

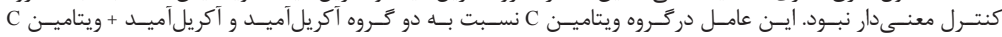

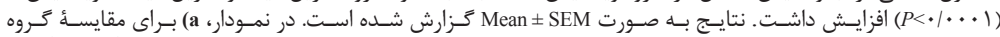

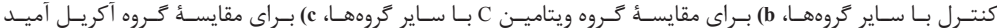

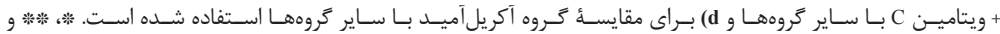

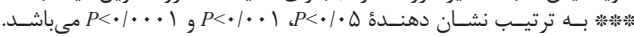

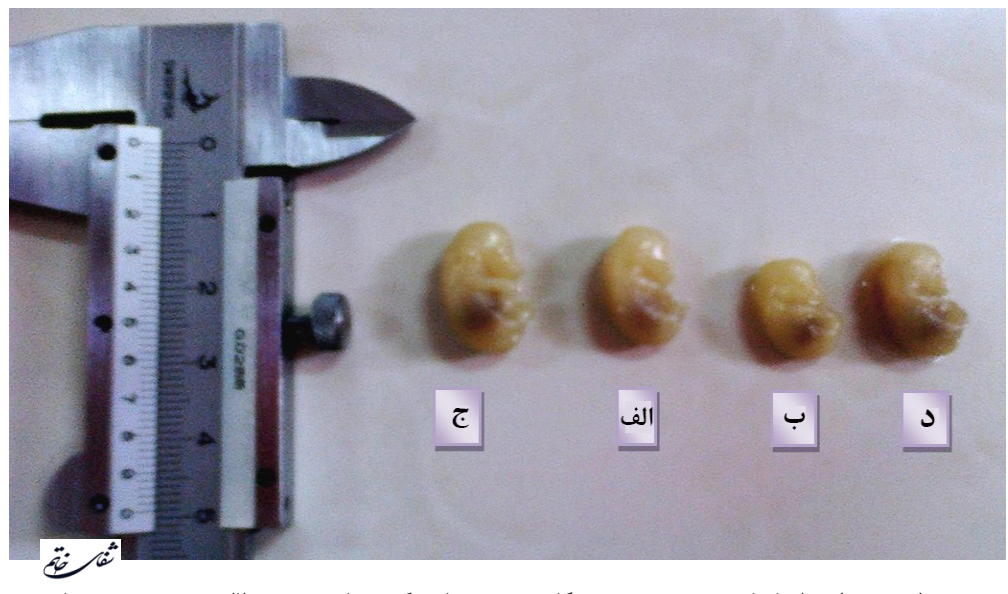

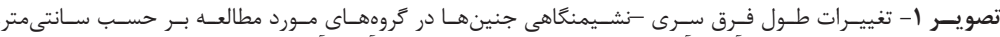

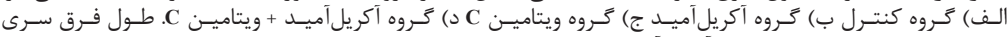

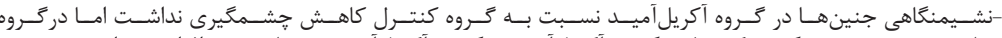

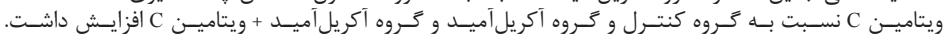

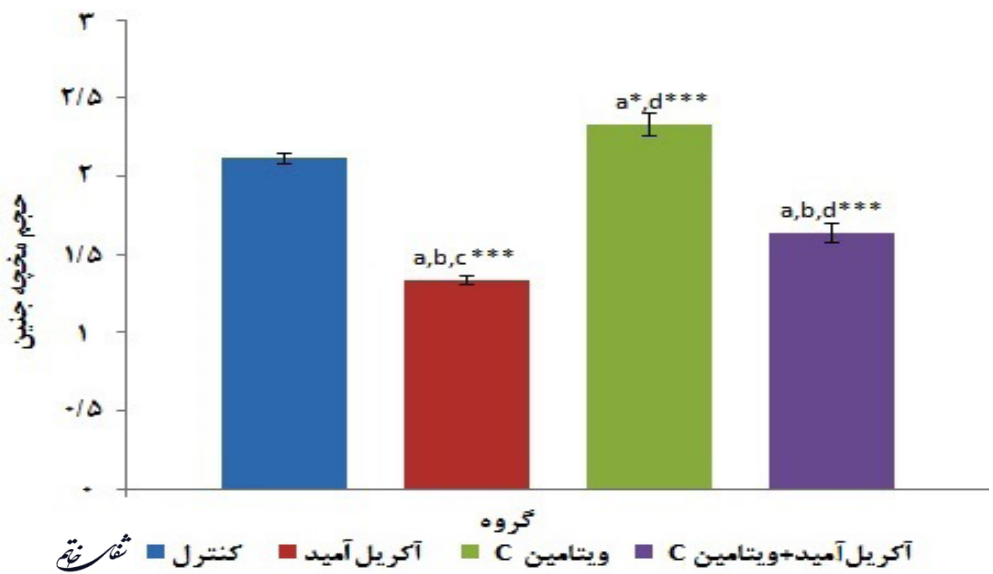

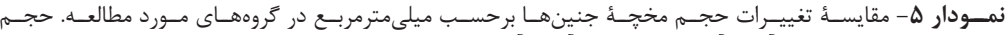

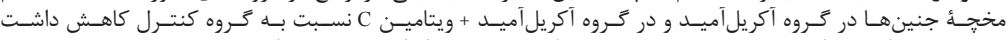
مخجا

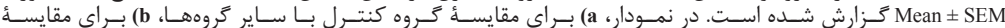

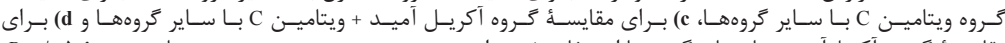

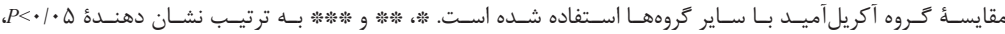

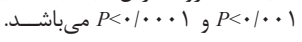




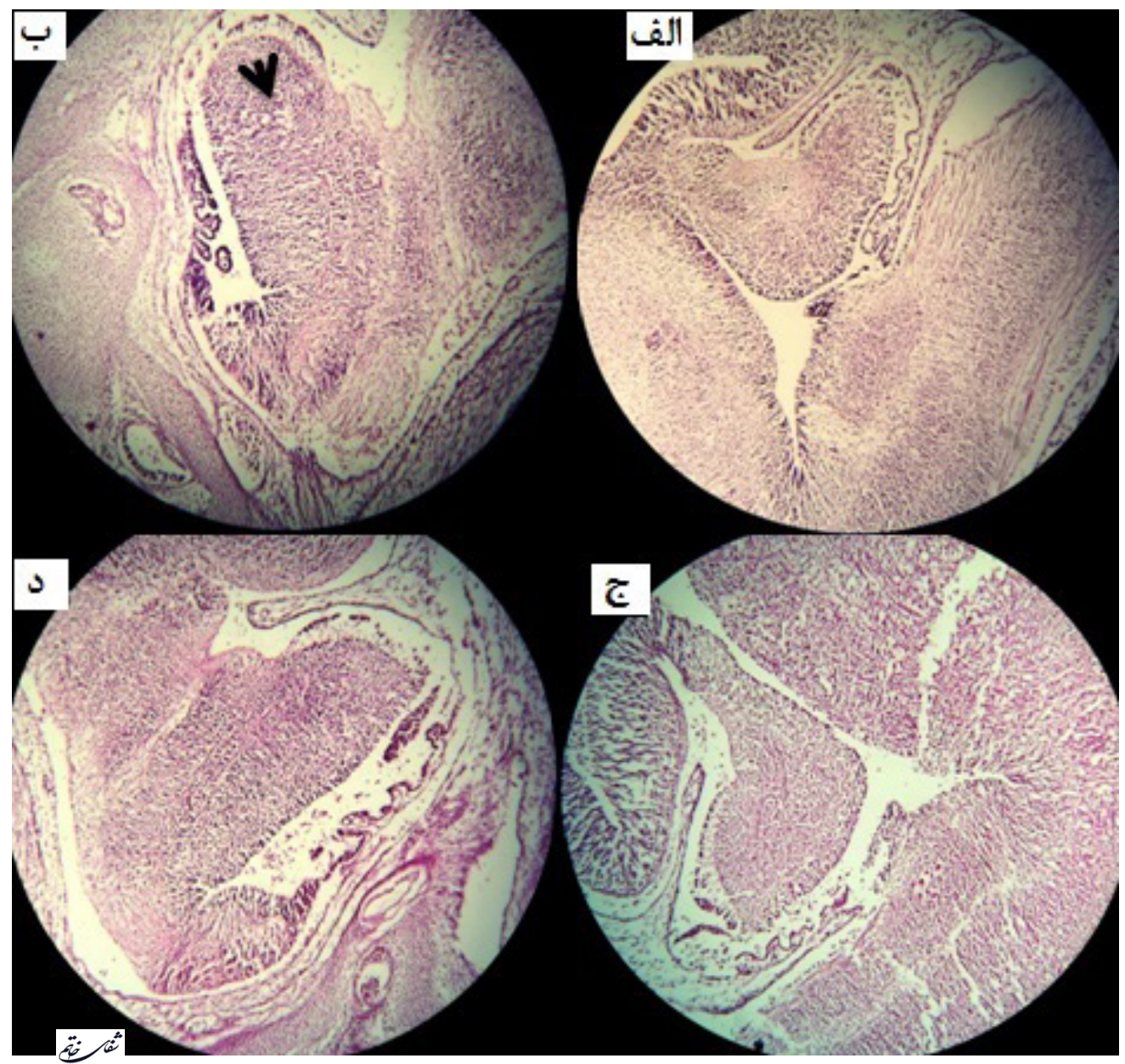

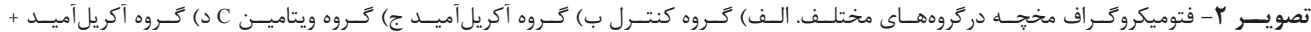

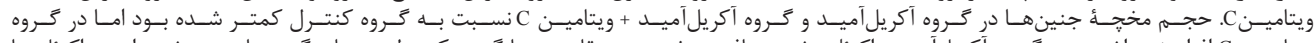

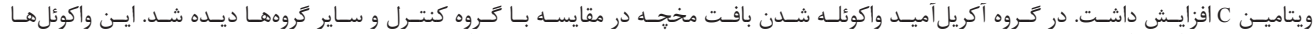

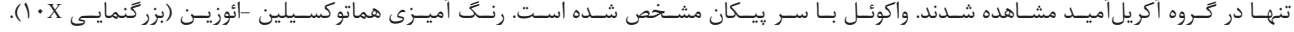

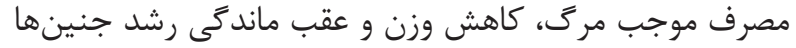

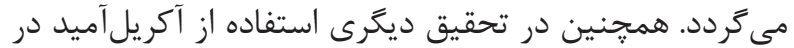

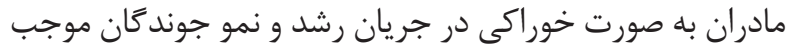

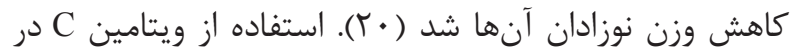

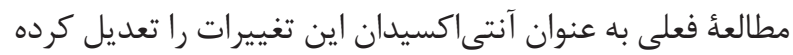

$$
\text { و كاهش مى مدهد. }
$$

مخجه نقش عمدهاى را در زمانبندى فعاليتهاى حر كتى برعهده

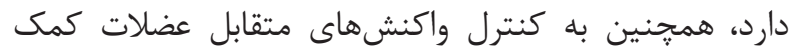

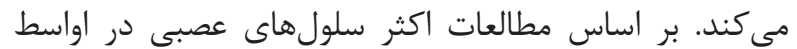

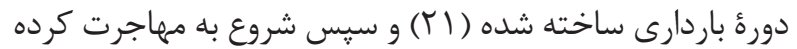

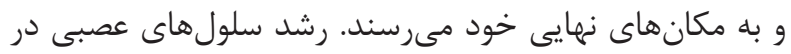

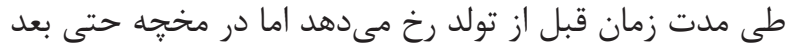
از تولد هم اتفاق مى افتد (YT) در اين مطالعه از روش استريولوزى و رنتَ آميزى هماتوكسيلين

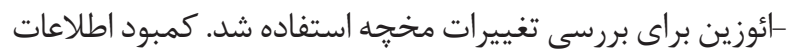

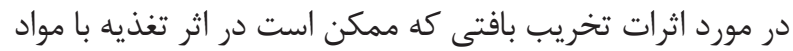

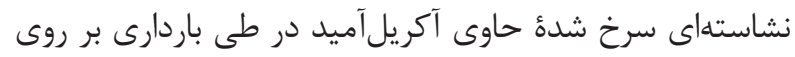
سيستم عصبى جنين ايجاد شود، هدف اين مطالعه قرار گرفت بارئ
همجنين در بررسى هاى بافتشناسى با رنتَآميزى هماتو كسيلين

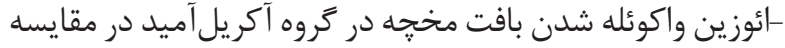

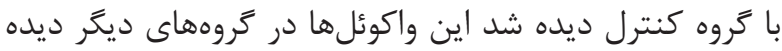

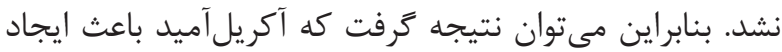

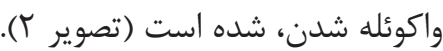

$$
\text { بحث و نتيجه كيرى }
$$

مطالعُ حاضر نشان داد تجويز آكريلآميد به موشهاى صحرايى

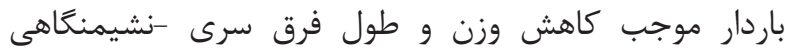

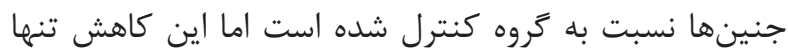

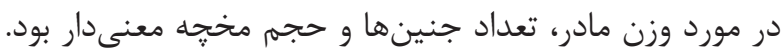

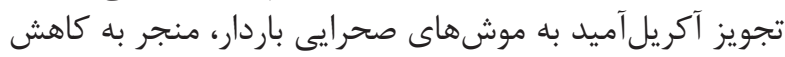

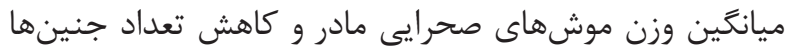

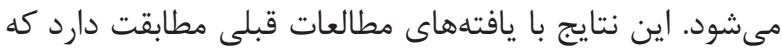

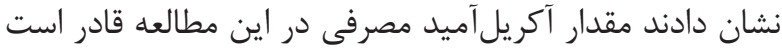

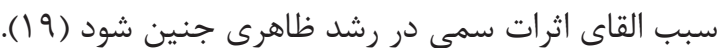
تحقيقات نشان مىدهد در معرض قرارگرفتن داخل رحمى

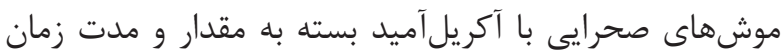


صحرايى نابالغ نر و ماده بررسى شده بود (Y9) و و واكوئوله شدن

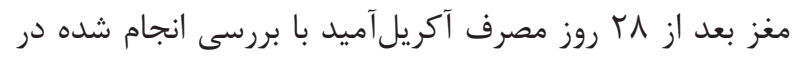

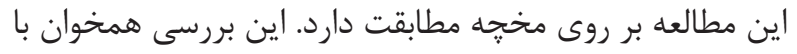

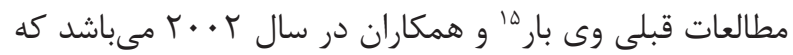

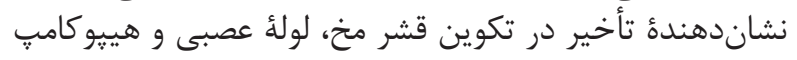

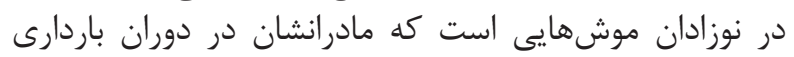

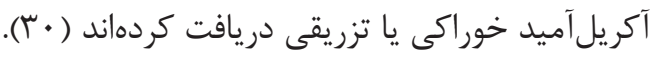

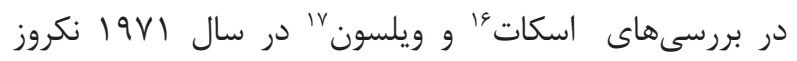

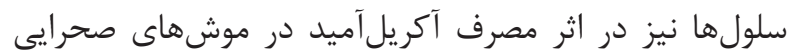

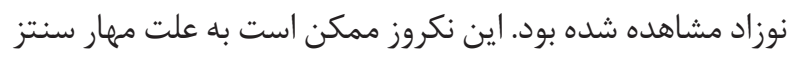

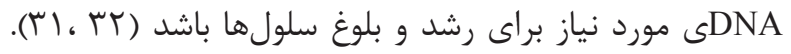

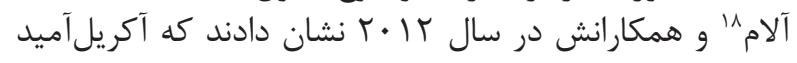

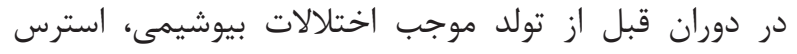

اكسيداتيو و القاى تغييرات ساختار مخحه مىشود (بسان).

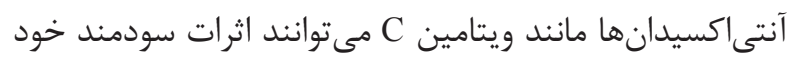

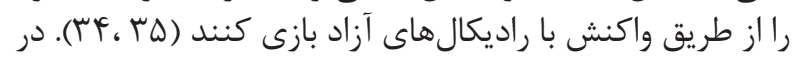

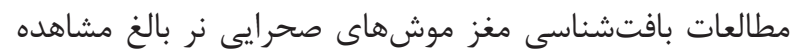

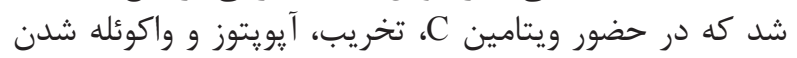

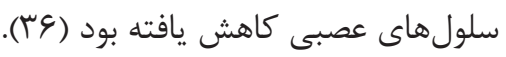

استفاده از ويتامين C در اين مطالعه نيز اثرات تخريبى و داهي

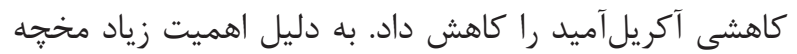

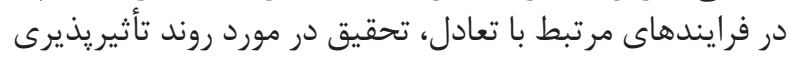

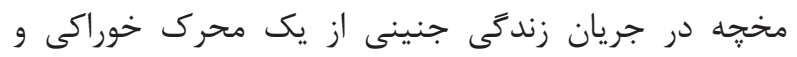

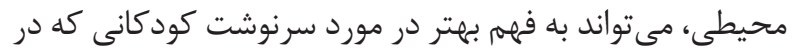

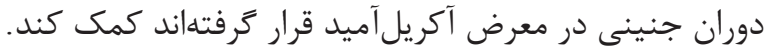

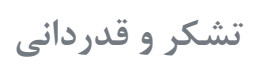

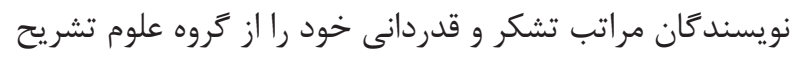

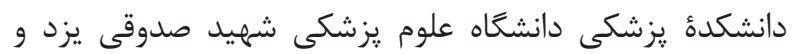
خانمها اقدس مير جليلى و راضيه كمالى اعلى اعلام مى دارند.

1. Namjoo A, Rafieian KM, Heidarian E. The Effect of acryl amide on tissue changes, blood and enzymatic parameters in male rats. J Shahrekord Univ Med Sc. 2013; 14(6): 27-37.

2. Thonning Olesen P, Olsen A, Frandsen H, Frederiksen K, Overvad K, Tjønneland A. Acrylamide exposure and incidence of breast cancer among postmenopausal women in the Danish Diet, Cancer and Health Study. Int J Cancer. 2008; 122(9): 2094-100.

3. Commission JFWCA, Programme JFWFS, Organization WH. Codex Alimentarius: Food hygiene, basic

\footnotetext{
${ }^{10}$ Central Nervous System

${ }^{11}$ Barber

${ }^{12}$ Lopachin

${ }^{13}$ Mottram

${ }^{14}$ Sayed

${ }^{15}$ Wei Bhaar
}

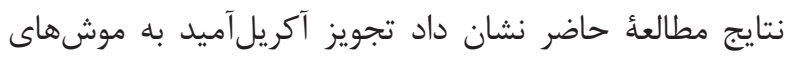

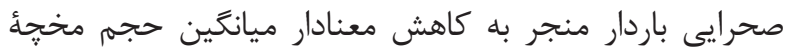

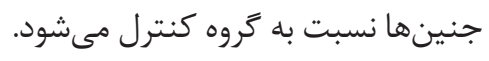

يافتههاى قبلى نشان دهندئ حساسيت بالاى مغز نوزادان

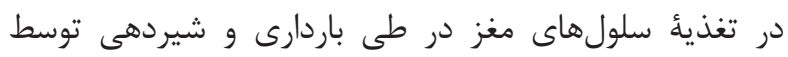

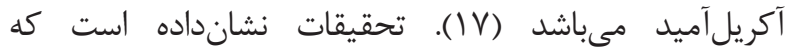

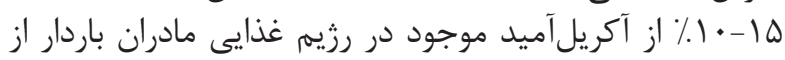

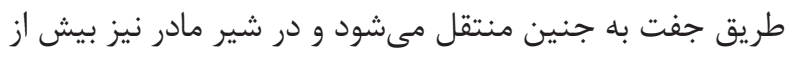

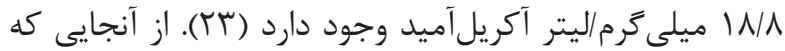

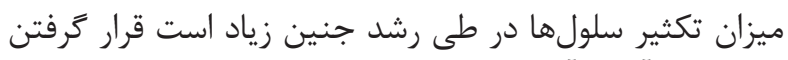

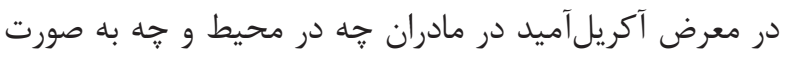

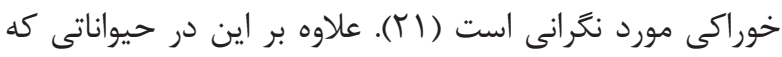

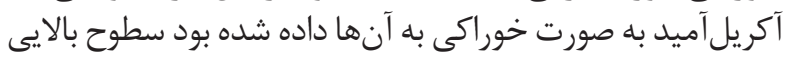

از همو كلوبين متصل شده با آكريل آميد وجود داشت داده (9 آدرد ).

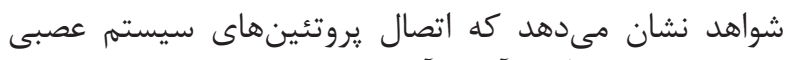

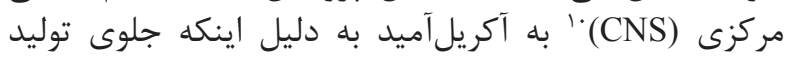

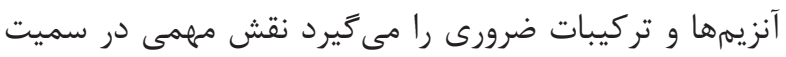

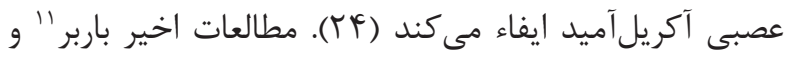

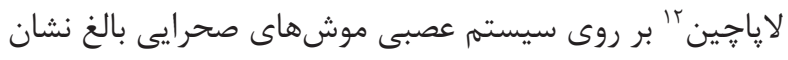

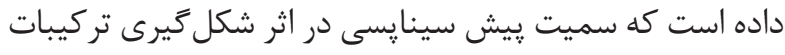

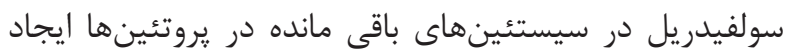

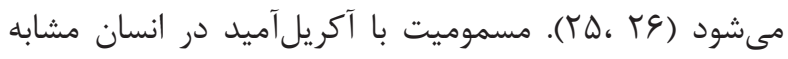

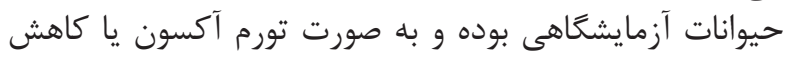
در تعداد و قطر آكسونها مشاهده مي شونود (TV).

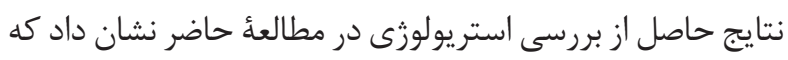

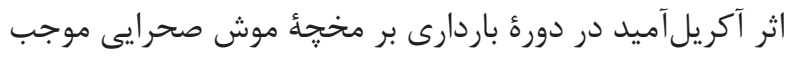

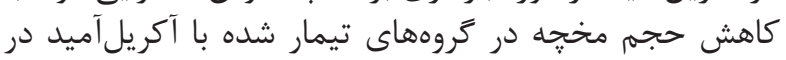

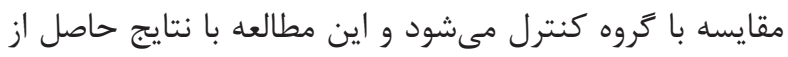

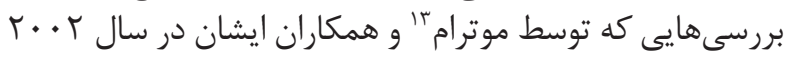

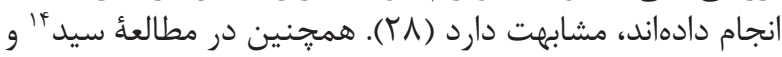

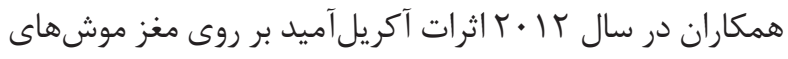

منابع

texts. 3rd ed. Food \& Agriculture Org. 2003; p. 57-63.

4. Lineback DR, Coughlin JR, Stadler RH. Acrylamide in foods: a review of the science and future considerations. Annu Rev Food Sci Technol. 2012; 3: 15-35.

5. Sabik L. Acrylamide-induced genotoxic, biochemical and pathological perturbations in male rats liver. J Am Sci. 2011; 7(1): 1092-6.

6. Alimentarius C. Joint FAO/WHO food standards programme. Codex committee on methods of analysis and sampling: 19th session, Budapest, Hungary, Criteria

$$
\begin{aligned}
& { }^{16} \text { Scott } \\
& { }^{17} \text { Wilson } \\
& { }^{18} \text { Allam }
\end{aligned}
$$


for evaluating acceptable methods for codex purposes. 1994; p. 342-68.

7. Ricaurte GA, Guillery R, Seiden L, Schuster C, Moore $\mathrm{R}$. Dopamine nerve terminal degeneration produced by high doses of methylamphetamine in the rat brain. Brain Res. 1982; 235(1): 93-103.

8. Silva C, Prediger RD, Rego AC, Ali SF, Ribeiro CAF. Disruption of striatal glutamatergic/GABAergic homeostasis following acute 2 methamphetamine in mice. Neurotoxicol Teratol. 2012; 34(5): 522-5.

9. Allam A, Abdul-Hamid M, Zohair K, Ajarem J, Allam G, El-Ghareeb A. Prenatal and perinatal acrylamide disrupts the development of cerebrum and medulla oblongata in albino rats. AFR J Biotechnol. 2012; 11(29): 7570-8.

10. Kim EJ, Won R, Sohn JH, Chung M, Nam TS, Lee HJ, et al. Anti-oxidant effect of ascorbic and dehydroascorbic acids in hippocampal slice culture. Biochem Biophys Res Commun. 2008; 366(1): 8-14.

11. Rice ME. Ascorbate regulation and its neuroprotective role in the brain. Trends Neurosci. 2000; 23(5): 209-16.

12. Grünewald R. Ascorbic acid in the brain. Brain Res Brain Res Rev. 1993; 18(1): 123-33.

13. Agus DB, Vera JC, Golde DW. Stromal cell oxidation a mechanism by which tumors obtain vitamin C. Cancer Res. 1999; 59(18): 4555-8.

14. Soujanya S, Lakshman M, Anand Kumar A. Histopathological and ultrastructural changes induced by imidacloprid in brain and protective role of vitamin C in rats. J Chem Pharma Res. 2012; 4(9): 4307-18.

15. Mayhew T. A review of recent advances in stereology for quantifying neural structure. J Neurocytol. 1992; 21(5): 313-28.

16. Schmitz C, Hof P. Design-based stereology in neuroscience. Neuroscience. 2005; 130(4): 813-31.

17. Garey J, Ferguson SA, Paule MG. Developmental and behavioral effects of acrylamide in Fischer 344 rats. Neurotoxicol Teratol. 2005; 27(4): 553-63.

18. Korbo L, Pakkenberg B, Ladefoged O, Gundersen HJ, Arlien-Søborg P, Pakkenberg H. An efficient method for estimating the total number of neurons in rat brain cortex. J Neurosci Methods. 1990; 31(2): 93-100.

19. Yaylayan VA, Stadler RH. Acrylamide formation in food: A mechanistic perspective. J AOAC Int. 2005;
88(1): 262-7.

20. Wang H, Huang P, Lie T, Li J, Hutz RJ, Li K, et al. Reproductive toxicity of acrylamide-treated male rats. Reprod Toxicol. 2010; 29(2): 225-30.

21. Schettgen T, Kütting B, Hornig M, Beckmann M, Weiss T, Drexler H, et al. Trans-placental exposure of neonates to acrylamide-a pilot study. Int Arch Occup Environ Health. 2004; 77(3): 213-6.

22. Waggas AM, Balawi E. Neurophysiological study on possible protective effect of rosemary (Rosmarinus officinalis) leaves extract in male albino rats treated with acrylamide. AEJSR. 2008; 3(2): 163-71.

23. Sörgel F, Weissenbacher R, Kinzig-Schippers M, Hofmann A, Illauer M, Skott A, et al. Acrylamide: increased concentrations in homemade food and first evidence of its variable absorption from food, variable metabolism and placental and breast milk transfer in humans. Chemotherapy. 2003; 48(6): 267-74.

24. Sakr SA, Badawy GM, El-Sayyad HI, Afify HS. Adverse effects of acrylamide on the developing retina of albino rats. J Basic Appl Sci Res. 2010; 1(7): 706-12.

25. Allam A, El-Ghareeb A, Abdul-Hamid M, Baikry A, Sabri M. Prenatal and perinatal acrylamide disrupts the development of cerebellum in rat: biochemical and morphological studies. Toxicol Ind Health. 2011; 10(3): 1814-25.

26. LoPachin RM, Gavin T, Geohagen BC, Das S. Neurotoxic mechanisms of electrophilic type-2 alkenes: soft-soft interactions described by quantum mechanical parameters. Toxicol Sci. 2007; 98(2): 561-70.

27. Edwards P, Gispen W. Peripheral pain fiber function is relatively insensitive to the neurotoxic actions of acrylamide in the rat. Toxicol Appl Pharmacol. 1991; 111(1): 43-8.

28. Millard JT, White MM. Diepoxybutane cross-links DNA at 5'-GNC sequences. Biochemistry. 1993; 32(8): 2120-4.

29. Sayed MR, Mohamed-Assem SM, Sohair RF, Salma AE-A. Hazardous effects of acrylamide on immature male and female rats. AJPP. 2012; 6(18): 1367-86.

30. Weisshaar R, Gutsche B. Formation of acrylamide in heated potato products-model experiments pointing to asparagine as precursor. DLR. 2002; 98(11): 397-400.

31. Krinke G, Classen W, Vidotto N, Suter E, Würmlin C. Detecting necrotic neurons with fluoro-jade stain. 


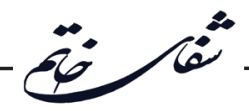

Exp Toxicol Pathol. 2001; 53(5): 365-72.

32. Beiswanger C, Mandella R, Graessle T, Reuhl K, Lowndes H. Synergistic neurotoxic effects of styrene oxide and acrylamide: glutathione-independent necrosis of cerebellar granule cells. Toxicol Appl Pharmacol. 1993; 118(2): 233-44.

33. Barber DS, LoPachin RM. Proteomic analysis of acrylamide-protein adduct formation in rat brain synaptosomes. Toxicol Appl Pharmacol. 2004; 201(2): 120-36.
34. Friedman M, Levin CE. Review of methods for the reduction of dietary content and toxicity of acrylamide. J Agric Food Chem. 2008; 56(15): 6113-40.

35. Anese M, Suman M, Nicoli MC. Acrylamide removal from heated foods. Food Chem. 2010; 119(2): 791-4.

36. Zama D, Meraihi Z, Tebibel S, Benayssa W, Benayache F, Benayache S, et al. Chlorpyrifos-induced oxidative stress and tissue damage in the liver, kidney, brain and fetus in pregnant rats: The protective role of the butanolic extract of Paronychia argentea L. Indian J Pharmacol. 2007; 39(3): 145-50. 